\title{
SyMmetrical Weighted SubSPACE Holistic APPROACH FOR EXPRESSION RECOGNITION
}

\author{
G.P.Hegde ${ }^{1}$, M. Seetha ${ }^{2}$ and Nagaratna Hegde ${ }^{3}$ \\ ${ }^{1}$ Department of Computer Science and Engineering, SDMIT, Ujire, VTU, Belgaum \\ ${ }^{2}$ Department of Computer Science and Engineering, GNITS, Hyderabad \\ ${ }^{3}$ Department of Computer Science and Engineering, VCE, Hyderabad
}

\begin{abstract}
Human face expression is one of the cognitive activity or attribute to deliver the opinions to others. This paper mainly delivers the performance of appearance based holistic approach subspace methods based on Principal Component Analysis (PCA). In this work texture features are extracted from face images using Gabor filter. It was observed that extracted texture feature vector space has higher dimensional and has more number of redundant contents. Hence training, testing and classification time becomes more. The expression recognition accuracy rate is also reduced. To overcome this problem Symmetrical Weighted 2DPCA (SW2DPCA) subspace method is introduced. Extracted feature vector space is projected in to subspace by using SW2DPCA method. By implementing weighted principles on odd and even symmetrical decomposition space of training samples sets proposed method have been formed. Conventional PCA and 2DPCA method yields less recognition rate due to larger variations in expressions and light due to more number of feature space redundant variants. Proposed SW2DPCA method optimizes this problem by reducing redundant contents and discarding unequal variants. In this work a well known JAFFE databases is used for experiments and tested with proposed SW2DPCA algorithm. From the experimental results it was found that facial recognition accuracy rate of GF+SW2DPCA based feature fusion subspace method has been increased to $95.24 \%$ compared to 2DPCA method.
\end{abstract}

\section{KEYWORDS}

Subspace, Gabor filter, Expression recognition, Symmetrical weight, Feature extraction, Classifier

\section{INTRODUCTION}

Facial expression exhibits the emotional state of a human being immediately as good means of communication. Hence through different expressions person can get interacted with each other and can understand their intensions and opinions [1-3]. In driving the vehicle, driver may get fatigue condition. This can be detected by facial expression recognition system. In security fields, expression recognition much needed for controlling the criminal people by identifying their expressions. While playing the games, according to face expressions the game will be controlled. Mind tempering needs face expression recognition. Person can interact with computers by exhibiting expressions. Different approaches were analysed by several authors. Still subspace based approaches finds large applications for dimensional and redundant data reduction. Appearance based and geometrical based subspace approaches are most common. [4][5][6]. Subspace methods finds major role during dimension reduction, redundant contents removing and feature extraction of face images. Sometimes direct implementation of subspace methods can reduce the recognition rate during larger facial expressions, larger variations of light and face poses. Hence it is better to use feature extraction approach and then removing the redundant contents from feature vector space using subspace methods is still more robust. Different work has been carried out for extraction of face features and classification of expressions. Ya Zheng, Xiuxin Chen, Chongchong Yu and Cheng Gao (2013) proposed fast local binary pattern method 
(FLBP) for dimensional reduction purpose [7]. They have shown FLBP uses fast PCA method and it reduces the feature vectors space in to subspace. They worked on LBP histogram to decrease the complexity of the algorithm. Yang Q. Ding, X. proposed symmetrical PCA for face recognition [8]. In this work, facial expression recognition algorithm based on feature extraction by Gabor filter (GF) and subspace projection by SW2DPCA method is introduced.

\section{BRIEF OVERVIEW OF SUbSPACE MethodS}

In subspace models un-correlation leads to increase in recognition rate by reducing redundancy. Subspace methods that introduce redundancy removal, feature extraction and low-dimensional feature representation of face objects with enhanced discriminatory power. Data reduction, redundant content removing and extraction of features are used to solve the problem of poor facial expression recognition at certain extent by improving the accuracy [9][10]. Process of reducing the number of variables in larger set of database is one of the properties of subspace analysis [9],[10]-[16]. In the context of face recognition, faces images are represented as highdimensional pixel arrays. Time and space complexities are two main problems for certain task in image analysis. Literature survey is carried out on various linear PCA based subspace methods. Principal Component Analysis (PCA) [9-11], CPCA [16], PC ${ }^{2}$ A [17], SPCA [19], 2DPCA [20], 2D ${ }^{2}$ PCA [21-22], Enhance PC ${ }^{2} A$ [21]. State of the art chart is given in table 2.

\section{Proposed Method}

The main objective of this work is to convert extracted feature vector space into subspace using suitable PCA based method by discarding the redundant contents at certain extent from the set of images. The conventional PCA and 2DPCA does not account for efficient recognition due to more number of principal variants. 2DPCA method considers a 2D image as a matrix rather than 1D image n-vectors [22]. In this work Symmetrical Weighted 2D Principal Component Analysis (SW2DPCA) method is proposed. Initially Gabor filter bank $(5 \times 8=40)$ has been formed. It was found that extracted feature vector space has larger dimensional area. This causes the redundant variants coefficients and reduces the recognition rate and increases the training time and classification time. By observing all these drawbacks high dimensional space has been reduced by implementing SW2DPCA method. Once PCA method have been applied to image sets it was found that principal eigen components are having higher variations and unequal distribution of variants. This problem would causes low expression recognition rate. All principle components are having larger variations of components this can be reduced and made equal by implementing weighted principal components analysis.

Total dimension of the image is given by $\mathrm{N}=\mathrm{pxq}$. The basic difference between PCA and 2DPCA is in 2DPCA each column of input 2D image a different sample. Thus q such p-vectors from each of the $\mathrm{M}$ images are used to build the covariance matrix. The number of the principal components in the 2DPCA cannot exceed $\mathrm{q}$ whereas the number $\mathrm{k}$ of their conventional counterpart is bounded with the total number $\mathrm{M}$ of the training samples (typically $\mathrm{q}>N$ ). Consider $2 \mathrm{D}$ training set as $I_{i}=\left[I_{1}, I_{2}, \ldots \ldots . I_{M}\right]$ of image vectors of size NxM. Where number of samples are given as M. Then compute the mean value of the training set image vectors at each pixel level called mean face. Size of the mean face is given as

$$
\overline{I_{i}}=\frac{1}{M} \sum_{i=1}^{M} I_{i}
$$

Then subtract the mean value from image $I_{\mathrm{i}}$ and compute mean subtracted image such as

$$
\Psi_{i}=\left(I_{i}-\overline{I_{i}}\right)
$$




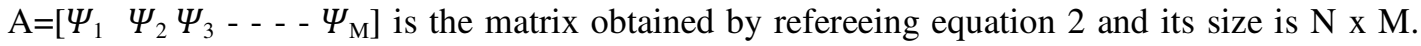
Then covariance matrix is computed which represents the scatter degree of all feature vectors related to the mean vector.

$$
C=A \cdot A^{T}=\frac{1}{M} \sum_{i=1}^{M} \Psi_{i} \Psi_{i}^{T}
$$

From the NXN covariance matrix eigenvectors are calculated, the size of the eigenvectors is considered as M x M. Covariance matrix can be computed as

$$
L=A \cdot A^{T}=\frac{1}{M} \sum_{i=1}^{M} \Psi_{i} \Psi_{i}^{T}
$$

In digital signal processing even and odd decomposition, can carried out by breaking a signal into two component signals, one is even symmetry signal and the other is odd symmetry signal. If a mirror image with $S / 2$ for signal then it is treated as even symmetrical. That is, sample $x[S / 2+1]$ must equal $x[S / 2-1]$, sample $x[S / 2+2]$ must equal $x[S / 2-2]$, etc. Similarly, odd symmetry occurs when the matching image feature points have equal magnitudes but are opposite in sign, such as: $x[S / 2+1]=-x[S / 2-1], x[S / 2+2]=-x[S / 2-2]$, etc. Let us consider $f(x)$ be a non-zero, real-valued function whose domain is symmetric about the origin; that is, $\mathrm{f}(\mathrm{x})$ exists implies $\mathrm{f}(-\mathrm{x})$ exists. Then $\mathrm{f}(\mathrm{x})$ can be uniquely expressed as the sum of an even function and an odd function.

Algebraically, a function is even if $\mathrm{f}(-\mathrm{x})=\mathrm{f}(\mathrm{x})$, and this condition manifests itself geometrically as symmetry with respect to the $y$-axis in the graph of $y=f(x)$. A function is odd if $f(-x)=-f(x)$, and geometrically this means that the graph of $\mathrm{y}=\mathrm{f}(\mathrm{x})$ is symmetric with respect to the origin. For any function if $f(x)$ is not equal to zero then we define the functions $f_{o}(x)=[f(x)-f(-x)] / 2$ and $f_{e}(x)=[f(x)+f(-x)] / 2$.

This odd-even rule can be applied to decomposition of face images. Our training set images are $I_{i}=\left[I_{1},=I_{2}, \ldots . I_{M}\right]$ mirror symmetrical training image set is $I_{i}^{S}=\left[I_{1}^{S}, I_{2}^{S} \ldots . . I_{M}^{S}\right]$, So the $\mathrm{i}^{\text {th }}$ image can be decomposed as $I_{i}=I_{o i}+I_{e i}$. Where odd symmetrical image can be denoted by $I_{o i}=\left(I_{i}-I_{i}^{M}\right) / 2$ and even symmetrical image can be denoted as $I_{e i}=\left(I_{i}+I_{i}^{M}\right)$. Here $\mathrm{i}=1,2,3, \ldots .$. ... Odd symmetrical sample set $\left(\mathrm{I}_{\mathrm{o} 1}, \mathrm{I}_{\mathrm{o} 2}, \mathrm{I}_{03}, \ldots, \mathrm{I}_{\mathrm{oM}}\right)$ and even symmetrical sample $\left(\mathrm{I}_{\mathrm{e} 1}, \mathrm{I}_{\mathrm{e} 2}, \mathrm{I}_{\mathrm{e} 3}, \ldots . \mathrm{I}_{\mathrm{eM}}\right)$ set both are derived from original training sample set by mirror symmetrical transform. The total scatter matrix of the three samples that is original, odd and even set can be defined as

$$
\begin{gathered}
S_{T}=\sum_{i=1}^{M} \Psi_{i} \Psi_{i}^{T} \\
S_{o w}=\sum_{i=1}^{M} \Psi_{e i} \Psi_{i}^{T} \\
S_{e w}=\sum_{i=1}^{M} \Psi_{e i} \Psi_{e i}^{T}
\end{gathered}
$$

Where, $S_{\mathrm{T}}=\mathrm{S}_{\mathrm{oi}}+\mathrm{S}_{\mathrm{ei}}$, hence the eigen value decomposition on $\mathrm{S}_{\mathrm{T}}$ is equal to the eigen decomposition on $S_{\mathrm{oi}}$ and $\mathrm{S}_{\mathrm{ei}}$. Hence, image $\mathrm{I}_{\mathrm{i}}$ can be reconstructed by the feature vector of $\mathrm{S}_{\mathrm{oi}}$ and $S_{\mathrm{ei}}$. With respect to eigen theory assume all the non-zero eigen values of $S_{\mathrm{oi}}$ and $S_{\mathrm{ei}}$ are $\lambda_{\mathrm{oi}}$ and $\lambda_{\mathrm{ej}}$. and the corresponding eigen vectors are $\mathrm{w}_{\mathrm{oi}}$ and $\mathrm{w}_{\mathrm{ej}}$. Where $\mathrm{i}=1 \ldots$ rank $\left(\mathrm{S}_{\mathrm{oT}}\right)$ and $\mathrm{j}=1 \ldots$ 
$\operatorname{rank}\left(\mathrm{S}_{\mathrm{eT}}\right)$. Transformation of weight matrix for odd $\left(\Omega_{\mathrm{o}}\right)$ and even $\left(\Omega_{\mathrm{e}}\right)$ symmetrical sample sets be derived from above demonstration as

$$
\begin{gathered}
\Omega_{o}=\left[w_{o 1}, w_{o 2} \ldots \ldots \ldots w_{o_{r o}}\right], \Lambda_{o}=\operatorname{diag}\left(\lambda_{o 1}, \lambda_{o 2}, \lambda_{o 3} \ldots \ldots \lambda_{o r_{o}}\right) \\
\Omega_{e}=\left[w_{e 1}, w_{e 2} \ldots \ldots \ldots w_{e_{r e}}\right], \Lambda_{e}=\operatorname{diag}\left(\lambda_{e 1}, \lambda_{e 2}, \lambda_{e 3} \ldots \ldots \lambda_{e r_{e}}\right)
\end{gathered}
$$

Where $r_{0}=\operatorname{rank}\left(S_{\mathrm{OT}}\right), r_{\mathrm{e}}=\operatorname{rank}\left(\mathrm{S}_{\mathrm{eT}}\right)$

The representation of the odd and even symmetrical images can be represented as,

$$
I_{o i}=\Omega_{o} P_{o i}, P_{o i}=\Omega_{o}^{T} I_{o i}, I_{e i}=\Omega_{e} P_{e i}, P_{e i}=\Omega_{e}^{T} I_{e i}
$$

In above, $\mathrm{P}_{\mathrm{oi}}$ and $\mathrm{P}_{\mathrm{ei}}$ are the odd symmetrical feature and even symmetrical feature of the $\mathrm{i}^{\text {th }}$ face image. In order to reduce the effects made by the principal components which contain the variation due lighting or face expression, it can treat as each component equally and let each component have equal variance through transforming conventional PCA feature space to weighted PCA feature space by the following whitening transformation for odd symmetrical sample set and even symmetrical sample set:

$$
\begin{gathered}
Q_{o}=\Lambda_{o}^{-1 / 2} \Omega_{o}^{T}=\left(\lambda_{o 1}^{-1 / 2} w_{o 1}, \lambda_{o 2}^{-1 / 2} w_{o 2}, \ldots \ldots \ldots . \lambda_{o r_{o}}^{-1 / 2} w_{o r_{o}}\right) \\
Q_{o}=\Lambda_{e}^{-1 / 2} \Omega_{e}^{T}=\left(\lambda_{e 1}^{-1 / 2} w_{e 1}, \lambda_{e 2}^{-1 / 2} w_{e 2}, \ldots \ldots \ldots . \lambda_{e r_{e}}^{-1 / 2} w_{e}\right)
\end{gathered}
$$

Here $Q_{o}$ and $Q_{e}$ are the transform matrix of odd symmetrical images and even symmetrical images for W2DPCA feature space. In particular, the representation of the odd or even symmetrical images in W2DPCA feature space is

$$
\begin{gathered}
I_{o i}=Q_{o} z_{o i}, Z_{o i}=Q_{o}^{T} I_{o i}, \quad I_{e i}=Q_{e} z_{e i} z_{e i}=Q_{e}^{T} I_{e i} \\
z_{i}=\left[z_{e i}^{T}, z_{o i}^{T}\right]^{T}=\left[\begin{array}{c}
z_{e i} \\
z_{o i}
\end{array}\right], Q=\left[Q_{e}, Q_{o}\right], \Lambda=\operatorname{diag}\left[\Lambda_{e}, \Lambda_{o}\right] \\
I_{i}=Q z_{i}=\left[Q_{e}, Q_{o}\right]\left[\begin{array}{c}
z_{e i} \\
z_{o i}
\end{array}\right], z_{i}=Q^{T} I_{i}
\end{gathered}
$$

For feature selection in SWPCA [24], all the eigenvectors are ordered according to eigenvalues, and then select eigenvectors corresponding to first $\mathrm{m}$ largest eigenvalues. Since the variance (corresponding to eigenvalues) of the weighted even symmetrical components is bigger than the variance of the correlative components of weighted odd symmetrical components. So it is natural to consider the even symmetrical components first, and then the odd symmetrical components if necessary otherwise discarded. Expression recognition system using proposed method is shown in figure 1.

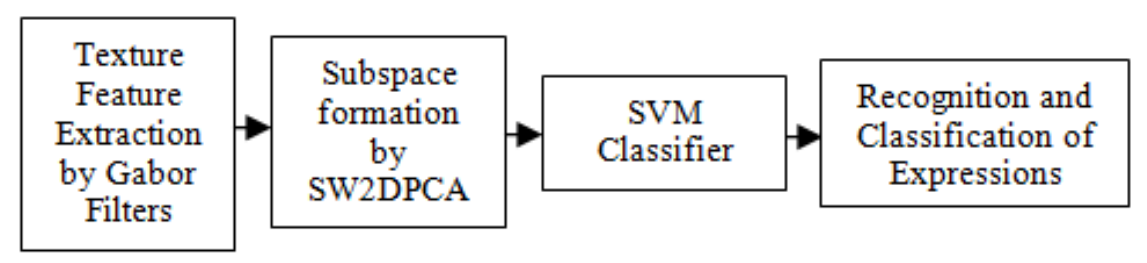

Figure 1. Block diagram of proposed system for recognition of expression 


\section{Brief About Gabor Filter And Classifier}

\subsection{Gabor Filter}

In this paper feature extraction by Gabor filter is not focused. Holistic texture features of a face image can be extracted using Gabor wavelets or Gabor filters (GF) [25]. In this work pahse part and magnitude parts are extracted using Gabor filter design of 40. This filter bank utilizes the group of wavelets and creates magnitude and phase parts at a specific frequency and specific orientation. These properties of Gabor filter are useful for texture analysis. Design of Gabor filters is followed in [26-30]. It is found that the Gabor feature vector space has high dimensional space and has more number of redundant coefficients. For the global Gabor filter bank with all the $m$ frequencies and $n$ orientations, denoted it as $\mathrm{G}(\mathrm{mxn})$. Yi-Chun Lee and Chin-Hsing Chen [31] have proposed feature extraction for face recognition based on Gabor filters and twodimensional locality preserving projections. Gabor wavelets have been successfully and widely applied to face recognition [32-33], face detection [34], texture segmentation [35] fingerprint recognition [36].

\subsection{Classifier}

After texture information is extracted from an image using Gabor filters it is normally encoded into a feature vector space. Given two subspace feature vectors, $W_{i}$ and $W_{r}$, a distance function computes the difference between them. The difference value which measures the accurately of the dissimilarity between the images from which the features were extracted. For larger distance, similarity values decreases. This Manhattan distance also called $L_{1}$ norm or city-block metric. The Manhattan distance [37] between train and test image feature vectors is given by,

$$
d_{M}=\left(W_{i}, W_{r}\right)=\sum_{i=1}^{i=M}\left|W_{r}[i]-W_{i}[i]\right|
$$

Here $W_{\mathrm{i}}$ is a vector describing the $i^{\text {th }}$ face class of training images. If $d_{M}$ is less than some predefined threshold value $\theta i$ then images are classified in to respective classes of expressions. Based on subspace projection coefficients of Gabor filter feature vector coefficients, all seven expression are classified using SVM classifier and recognition of images is achieved using Manhattan distance matching score values. We perform the facial expression recognition by using a support vector machine (SVM) [38] to evaluate the performance of the proposed method. SVM is a supervised machine learning technique that implicitly maps the data into a higher dimensional feature space. Consequently, it finds a linear hyper plane, with a maximal margin, to separate the data in different classes in this higher dimensional space. Given training set of $M$ labelled examples $T=\left\{\left(x_{i} y_{i}\right) \mid i=1, \ldots M\right\}$, where $x_{i} \in R^{n}$ and $y_{i} \in\{-1,1\}$, the test is classified by

$$
f(x)=\operatorname{sign}\left(\sum_{i=1}^{M} a_{i} y_{i} K\left(x_{i} x\right)+b\right)
$$

Where $a_{i}$ is Lagrange multipliers of dual optimization problem $\mathrm{b}$ is a bias and $K$ is a kernel function. Note that SVM allows domain specific selection of the kernel function. Although many kernels have been proposed, the most frequently used kernel function is the linear, polynomial, and radial basis function (RBF) kernels. In this study SVM+RBF method is used. 


\section{TESTing AND ReSult ANALYSIS}

\subsection{Pre-processing}

In this work, Japanese Female Facial Expression (JAFFE) [39] database is used for experiment. This database contains 213 images of 7 facial expressions ( 6 basic facial expressions +1 neutral) posed by 10 Japanese female models of 256x256 resolution. Each image has been rated on 6 emotion adjectives by 60 Japanese subjects. All the images of this database were pre-processed to obtain pure facial expression images, which have normalized intensity, uniform size and shape. The pre-processing procedure used in this work performs detecting facial feature points manually including eyes, nose and mouth, rotating to line up the eye coordinates, locating and cropping the face region using a rectangle according to face cropping model [40] as shown in figure 2 (a). Consider $d$ is the distance between two eyries as shown in fig 2a. then a rectangle is formed with respect to $\mathrm{d}$ is $2.2 \mathrm{~d} \times 1.8 \mathrm{~d}$; then images are scale down fixed size of $128 \times 96$, locating the centre position of the two eyes to a fixed position. Finally using a histogram equalization method illumination effects were removed. Fig. 2 (b) shows some examples of pure facial expression images after pre-processing from JAFFE database.

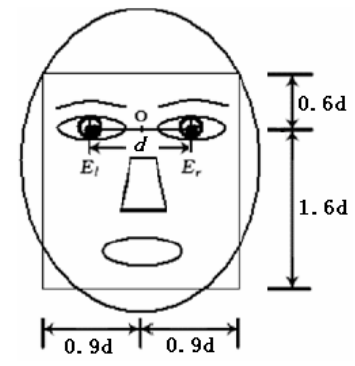

(a)

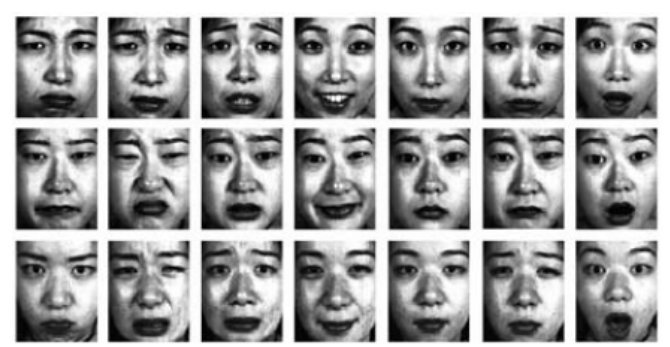

(b)

Figure 2. (a) Face cropping model (b) Few samples of cropped images of JAFFE database

\subsection{Testing and Analysis of Results}

The database is tested with 2DPCA and SW2DPCA methods by projecting the feature space of Gabor filter. Support Vector Machine Classifier (SVM) using RBF kernel method is used to classify the seven expressions of JAFFE database. To create SVM model, all 210 images of JAFFE database are considered. In that $70 \%$ of images are considered for training and $30 \%$ images are considered for testing using hold out cross validation classification method. The database is tested with all the subspace models and proposed approach. In addition to a drastic reduction in the number of coefficients, it is observed that a considerable improvement in the recognition rate relative to the facial expression recognition experiment.

Table 1. Gabor Feature dimension after and before implementing SW2DPCA

\begin{tabular}{|c|c|c|c|c|}
\hline $\begin{array}{c}\text { Gabor filter } \\
\text { bank }\end{array}$ & $\begin{array}{c}\text { Cropped } \\
\text { image } \\
\text { dimension }\end{array}$ & $\begin{array}{c}\text { Number of } \\
\text { Filters }\end{array}$ & $\begin{array}{c}\text { Original } \\
\text { dimension }\end{array}$ & $\begin{array}{c}\text { Feature } \\
\text { reduction } \\
\text { dimension }\end{array}$ \\
\hline $\mathrm{G}(5 \mathrm{x} 8)$ & $128 \mathrm{x} 96$ & 40 & 491520 & 3840 \\
\hline
\end{tabular}


International Journal of Computer Science \& Information Technology (IJCSIT) Vol 7, No 4, August 2015

Table 2. Brief about state of art

\begin{tabular}{|l|l|}
\hline Subspace Methods & FERR \\
\hline PCA [43] & $91.00 \%$ \\
\hline 2DPCA [44] & $78.00 \%$ \\
\hline 2DPCA + features[44] & $94.00 \%$ \\
\hline 2D PCA +features[44] & $84.50 \%$ \\
\hline Log Gabor [45] & $91.80 \%$ \\
\hline Gabor Filter (GF) [7] & $89.98 \%$ \\
\hline GF+PCA [1 ] & $81.70 \%$ \\
\hline DCT+PCA+RBF[42] & $90.50 \%$ \\
\hline GF+PCA+PNN[42] & $94.72 \%$ \\
\hline GF+PCA+RBF[42] & $94.11 \%$ \\
\hline
\end{tabular}

Table 3. Comparison of facial expression recognition rate (FERR) for JAFFE database

\begin{tabular}{|l|l|l|}
\hline $\begin{array}{c}\text { PCA based subspace } \\
\text { methods }\end{array}$ & \multicolumn{1}{|c|}{$\begin{array}{c}\text { FERR } \\
\text { in \% }\end{array}$} & $\begin{array}{c}\text { Classification Time in } \\
\text { Sec. }\end{array}$ \\
\hline GF+PCA[1] & $81.70 \%$ & -------- \\
\hline GF+2DPCA & $93.33 \%$ & 0.660622 \\
\hline GF+SW2DPCA & $95.24 \%$ & 0.655970 \\
\hline
\end{tabular}

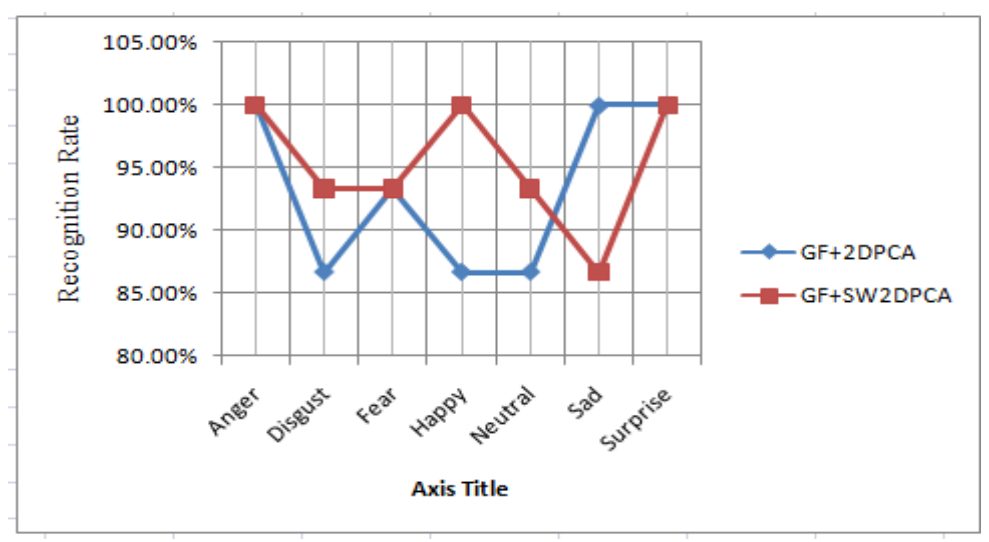

Figure 3. Comparison of expression rates for GF+2DPCA and GF+SW2DPCA 


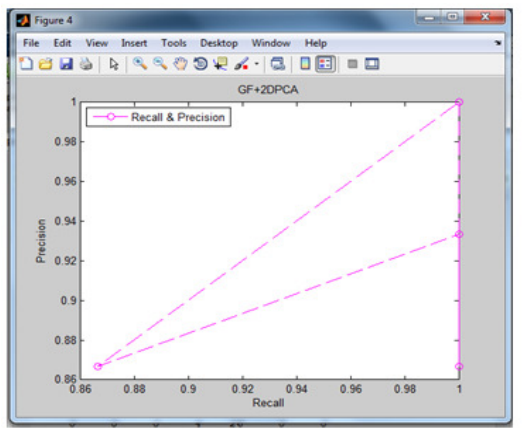

(a)

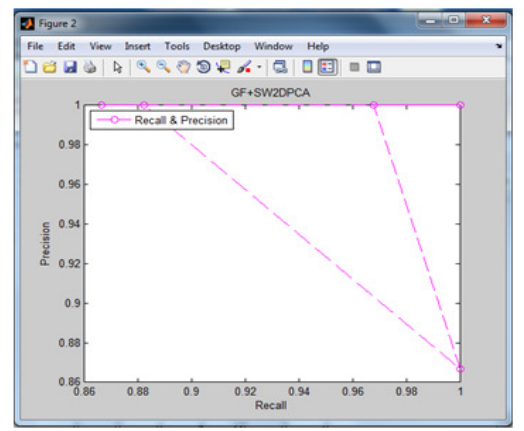

(b)

Figure 4. Precision and recall rates (a) GF+2DPCA, (b) GF+SW2DPCA method

Experimental results shows that proposed SW2DPCA method improves the average recognition accuracy rate by reducing redundant variants with odd /even symmetrical decomposition of input images. Gabor filtered image feature vectors space is projected by SW2DPCA method, hence original dimension of feature vector space is reduced to 3840 as given in table 1. Overall accuracy of facial expression rate is found to be $95.24 \%$ for pre-processed images as shown in table 3 . Among seven expressions three expressions were found to be $100 \%$ accuracy. Disgust and neutral, expressions accuracy rate is more in SW2DPCA method. Classification accuracy rate is better in SW2DPCA compare to 2DPCA method as shown in figure 3. The percentage of precision rate is given by ratio between number of images correctly recognized and total number of recognition for each person faces. Similarly, the percentage of recall rate is defined as ratio between numbers of correct images is recognized to total number of input faces for each person. Figure 4 illustrates the correct and wrong recognition of expressions. Classification rate of seven expressions of JAFFE database also improved. Experiments are performed using MATLAB, R2013a,version.

\section{CONCluSions}

Facial expression recognition using appearance based methods has significant application in different fields. Subspace based methods were already implemented for face recognition since from decade. In this paper a holistic texture feature extraction by Gabor filter and extracted feature vector space dimension is reduced by SW2DPCA subspace method is proposed. This proposed method performs well compared to other PCA and 2DPCA methods. All the second order principal components of SWPCA was made to equal by minimizing the redundant contents hence it improves the recognition efficiency at certain extent compare to state of art methods as mentioned in this paper even in larger variations expressions. Experimental results conclude that overall accuracy has been increased to $95.48 \%$.All seven expressions are classified using support vector machine classifier with radial basis function (SVM+RBF). Except sad expression of JAFFE database all other six expressions accuracy rate is increased in SW2DPCA method compared to 2DPCA method.

\section{ACKNOWLEDGEMENTS}

This work is supported by Dr. M, Seetha, Professor, Department of Computer Science and Engineering G. N. Institute of Technology and Science, Hyderabad, India, and also I thank for providing MATALAB tool 2013 version by Dr. Nagaratna Hegde, Professor, Department of Computer Science and Engineering, Vasavi College of Engineering, Hyderabad. 
International Journal of Computer Science \& Information Technology (IJCSIT) Vol 7, No 4, August 2015

\section{REFERENCES}

[1] Bettadapura V., "Face Expression Recognition and Analysis: The State of the Art," in Proceedings of IEEE Transaction on Pattern Analysis and Machine Intelligence, vol. 22, pp.1424-1445, 2002.

[2] C. Shan, S. Gong, and P. McOwan, "Robust facial expression recognition using local binary patterns," in proceedings of the IEEE International Conference Image Processing, pp. 914-17, 2005.

[3] Y. Tian, T. Kanade, and J.F. Cohn, "Facial Expression Analysis: Handbook of Face Recognition," New York City: Springer; 2003.

[4] Hsieh C., Lai S., and Chen Y., "An Optical Flow Based Approach to Robust Face Recognition under Expression Variations,” IEEE Transaction on Image Processing, vol. 19, no. 1, pp. 233-240, Jan 2010.

[5] Ya Zheng, Xiuxin Chen, Chongchong Yu and Cheng Gao Facial Expression Feature Extraction Based on FastLBP Journal of Software Vol. 8, No. 11, November 2013.

[6] Cruz, A.C, Bhanu, B. Thakoor,N.S. "Vision and Attention Theory Based Sampling for Continuous Facial Emotion Recognition," IEEE Transaction on Affective Computing, Volume:5 Issue:4 , April 2014.

[7] M.S. Bartlett, G. Littlewort, M. Frank, C. Lainscsek I. Fasel, and J. Movellan, "Recognizing facial expression: Machine learning and application to spontaneous behavior," in IEEE Conference on Computer Vision and Pattern Recognition, Vol. 2, pp. 568-73, 2005.

[8] Yang Q, Ding X ,'Symmetrical PCA in face recognition. In Proc. IEEE International Conference on Image Processing, New York, USA.pp.97-100. 2002.

[9] S.Z. Li, A.K. Jain, Gregory Shakhnarovich and Baback Moghaddam "Handbook of Face Recognition, Face Recognition in Subspaces” Springer-Verlag London Limited 2011.

[10] Dimitris Bolis, Anastasios Maronidis, Anastasios Tefas, and Ioannis Pitas "Improving the Robustness of Subspace Learning Techniques for Facial Expression Recognition” ICANN 2010, Part I, LNCS 6352, pp. 470-479, 2010. Springer-Verlag Berlin Heidelberg 2010.

[11] Jamal Hussain Shah, Muhammad Sharif, Mudassar Raza, and Aisha Azeem "A Survey: Linear and Nonlinear PCA Based Face Recognition Techniques" The International Arab Journal of Information Technology, Vol. 10, No. 6, November 2013.

[12] A. Adeel Mohammed, M. A. Sid-Ahmed Rashid Minhas, and Q. M. Jonathan Wu, "A face portion based recognition system using multidimensional PCA," IEEE $54^{\text {th }}$ International Midwest Symposium on Circuits and Systems, pp. 1 - 4, August 2011.

[13] Abegaz, T., Adams J, Bryant, K., Dozier, G., Popplewell, K., Ricanek, K., Shelton, J., and Woodard, D.L., "Hybrid GAs for Eigen-based facial recognition," IEEE Workshop on Computational Intelligence in Biometrics and Identity Management, pp. 127 - 130, April 2011.

[14] Chengliang Wang, LibinLan, MinjieGu, and Yuwei Zhang, "Face Recognition Based on Principle Component Analysis and Support Vector Machine," $3^{\text {rd }}$ International Workshop on Intelligent Systems and Applications, pp. 1 - 4, May 2011.

[15] Guan-Chun Luh, "Face recognition using PCA based immune networks with single training sample per person," International Conference on Machine Learning and Cybernetics, vol. 4, pp. 1773 - 1779 , July 2011.

[16] Ran He, Wei-Shi Zheng, Bao-Gang Hu, Xiang-Wei Kong, “Two-Stage Nonnegative Sparse Representation for Large-Scale Face Recognition,” In: IEEE Trans. on Neural Networks and Learning Systems, vol. 24, no. 1, Jan. 2013.

[17] Bag Soumen, and Sanyal Goutam, "An Efficient Face Recognition Approach using PCA and Minimum Distance Classifier,” International Conference on Image Information Processing, pp. 1 - 6, November 2011.

[18] Guoxia Sun, Huiqiang Sun and Liangliang Zhang, "Face Recognition Based on Symmetrical Weighted PCA," International Conference on Computer Science and Service System, pp. 2249 2252, August 2011.

[19] M.Turk, A Pentland. Eigenfaces for recognition, Journal of Cognitive Neuroscience 3 (1) (1991) 71 86.

[20] Jainxin $\mathrm{Wu}$, Zhi-hua Zhou, Face recognition with one training image per person, Pattern Recognition Letters 23(2002) 1711-1719.

[21] Songcan Chen, Daoqiang Zhang, Zhi-Hua Zhou, Enhanced $\mathrm{PC}^{2} \mathrm{~A}$ for face recognition with one training image per person, Pattern Recognition Letters 25 (2004) 1171-1181.

[22] D. Zhang, Z. H. Zhou, 2D ${ }^{2}$ PCA: 2-directional 2 dimensional PCA for efficient face representation and recognition, Neurocomputing 69(1-3) 2005 224-231. 
[23] Ch.V. Ramireddy, K.V.Krishna Kishore," Facial Expression Classification using Kernel based PCA with Fused DCT and GWT Features," 2013 IEEE International Conference on Computational Intelligence and Computing Research.

[24] Guoxia Sun, Huiqiang Sun and Liangliang Zhang, "Face Recognition Based on Symmetrical Weighted PCA," International Conference on Computer Science and Service System, pp. 2249 2252, August 2011.

[25] T. P. Weldon, W. E. Higgins and D. F. Dunn, "Efficient Gabor Filter Design for Texture Segmentation," Pattern Recognition, Vol. 29, No. 12, 1996, pp. 2005-2015. doi:10.1016/S00313203(96)00047-7

[26] Porat M., Zeevi Y, "The generalized Gabor scheme of image representation in biological and machine vision",IEEE Transactions on Pattern Analysis and Machine Intelligence, 1988, 10(4), pp.452 - 468.

[27] T. P. Weldon, W. E. Higgins and D. F. Dunn, "Efficient Gabor Filter Design for Texture Segmentation," Pattern Recognition, Vol. 29, No. 12, 1996, pp. 2005-2015.

[28] Y. Hamamoto, S. Uchimura, M. Watanabe, T. Yasuda, Y. Mitani and S.Tomita, "A Gabor FilterBased Method for Recognizing Handwritten Numerals," Pattern Recogni-tion, Vol. 31, No. 4, 1998, pp. 395-400.

[29] C. J. Liu and H. Wechsler, "Independent Component Analysis of Gabor Features for Face Recognition," IEEE Transactions on Neural Networks, Vol. 14, No. 4, 2003, pp. 919-928.

[30] C. J. Liu and H. Wechsler, "Gabor Feature Based Classi- fication Using the Enhanced Fisher Linear Discriminant Model for Face Recognition," IEEE Transactions on Image Processing, Vol. 11, No. 4, 2002, pp. 467-476.

[31] Y.-C. Lee and C.-H. Chen, "Feature Extraction for Face Recognition Based on Gabor Filters and Two-Dimensional Locality Preserving Projections," Proceedings of the 5th IEEE Conference on Intelligent Information Hiding and Multimedia Signal Processing, Kyoto, 12-14 September 2009, pp. 106-109.

[32] L. Shen and L. Bai, "Face Recognition Based on Gabor Features Using Kernel Methods," Proceedings of the 6th IEEE Conference on Face and Gesture Recognition, Seoul, 17-19 May 2004, pp. 170-175. doi:10.1109/AFGR.2004.1301526

[33] S. F. Xie, S. G. Shan, X. L. Chen and J. Chen, "Fusing Local Patterns of Gabor Magnitude and Phase for Face Recognition," IEEE Transactions on Image Processing, Vol. 19, No. 5, 2010, pp. 1349-1361. doi:10.1109/TIP.2010.2041397

[34] X. H. Li, K.-M. Lam, L. S. Shen and J. L. Zhou, "Face Detection Using Simplified Gabor Features and Hierarchical Regions in a Cascade of Classifiers," Pattern Recognition Letters, Vol. 30, No. 8 , 2009, pp. 717-728. doi:10.1016/j.patrec.2009.03.006

[35] T. P. Weldon, W. E. Higgins and D. F. Dunn, "Efficient Gabor Filter Design for Texture Segmentation," Pattern Recognition, Vol. 29, No. 12, 1996, pp. 2005-2015. doi:10.1016/S00313203(96)00047-7

[36] C. J. Lee and S. D. Wang, "Fingerprint Feature Extraction Using Gabor Filters," Electronics Letters, Vol. 35, No. 4, 1999, pp. 288-290. doi:10.1049/el:19990213

[37] Vadivel,A K Majumdar, Shamik Sural, (2003), "Performance comparison of distance metrics in content-based Image retrieval applications", International Conference on Information Technology (CIT), Bhubaneswar, India, pp. 159-164

[38] K.I. Kim, K. Jung, S.H. Park, H.J. Kim, "Support Vector Machines for Texture Classification,” IEEE Transactions on Pattern Analysis and Machine Intelligence, vol. 24, no. 11, pp. 1542-1550, 2002

[39] http://www.kasrl.org/jaffe_info.html

[40] F. Y. Shih, C. Chuang, "Automatic extraction of head and face boundaries and facial features", Information Sciences, Vol. 158, 2004, pp.117-130.

[41] Cruz, A.C, Bhanu, B. Thakoor,N.S. "Vision and Attention Theory Based Sampling for Continuous Facial Emotion Recognition," IEEE Transaction on Affective Computing, Volume:5 Issue:4 , April 2014.

[42] Ch.V. Ramireddy, K.V.Krishna Kishore," Facial Expression Classification using Kernel based PCA with Fused DCT and GWT Features," 2013 IEEE International Conference on Computational Intelligence and Computing Research.

[43] Chen Juanjuan, Zhao Zheng, Sun Han, Zhang Gan," Facial Expression Recognition Based on PCA Reconstruction." The 5th International Conference on Computer Science \& Education Hefei, China. August 24-27, 2010.

[44] Luiz S. Oliveria, Alessandro L. Koerich, and Marcello Mansano, Alceu S. Britto, "2D Principal Component Analysis for face and facial expression recognition", published in CISE-13-3-Oliveira. May/June-2011.

[45] Amir Jamshidnezhad and Md Jan Nordin, "An adaptive learning model based genetic for facial expression recognition,” International Journal of the Physical Sciences Vol. 7(4), pp. 619-623, 23 January, 2012, ISSN 1992 -1950 @ 2012. 


\section{Authors}

First-Author received M. Tech. degree in Computer Science and Engineering from Visvesvaraya Technical University (VTU) Belgaum. India, in 2009-2010, and Pursuing Ph.D. from Visvesvaraya Technical University (VTU) Belgaum. From 1994 to 2009, he was Lecturer, and became Assistant Professor in 2010 in department of Computer Science and Engineering at the SDM Institute of Technology, Ujire, Mangalore, India. SDMIT is affiliated to VTU Belgaum, India. First-Author is the author of over 18 technical publications, proceedings. His research interests include Image processing, Computer Networks, Software engineering, Software architecture.

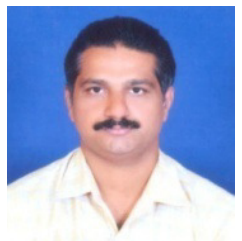

Second-Author received M.S. degree in Software System Engineering from the Birla Institute of Technology and Science (BITS), Pilani, India, and Ph.D. degree in Computer Science and Engineering from the Jawaharlal Neharu Technological University (JNTU) Hyderabad, India in 1999 and 2007, respectively. From 1999 to 2005, she was at the Computer Science Department of Chaitanya Bharathi Institute of Technology, Hyderabad. Assistant Professor and worked as Associate Professor in 2005 to 2008. She is currently a Professor in the Department of Computer Science and

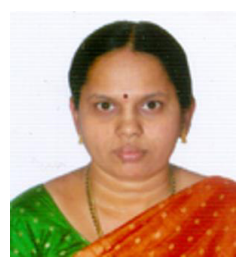
Engineering of G. Narayanamma Institute of Technology, Hyderabad and Sciences, affiliated to JNTU, Hyderabad, India. Second-author is the author of over 41 technical publications. Her interested areas are Image Processing, Pattern Recognition, Artificial Intelligence, Neural Networks, Data communication and Interfacing, Data mining and Computer Organization. Recognized as supervisor from Osmania University, Reviewer for International journals of Information Fusion, and ACM journal. 